\title{
nNOS $(+)$ striatal neurons, a subpopulation spared in Huntington's Disease, possess functional NMDA receptors but fail to generate mitochondrial ROS in response to an excitotoxic challenge
}

\section{Lorella M. T. Canzoniero ${ }^{1}$, Alberto Granzotto ${ }^{2}$, Dorothy M. Turetsky ${ }^{3}$, Dennis W. Choi ${ }^{4}$, Laura L. Dugan ${ }^{5,6}$ and Stefano L. Sensi ${ }^{2,78 *}$}

1 Department of Biological and Environmental Science, University of Sannio, Benevento, Italy

2 Molecular Neurology Unit, Center of Excellence on Aging, University "G. d'Annunzio," Chieti, Italy

${ }^{3}$ Department of Biochemistry and Microbiology, Oklahoma State University, Tulsa, OK, USA

${ }^{4}$ Department of Neurology, Stony Brook University, Stony Brook, NY, USA

${ }^{5}$ Department of Medicine, University of California San Diego, San Diego, CA, USA

${ }^{6}$ Department of Neuroscience, University of California San Diego, San Diego, CA, USA

7 Department of Neuroscience and Imaging, University "G. d'Annunzio," Chieti, Italy

${ }^{8}$ Departments of Neurology and Pharmacology, Institute for Memory Impairments and Neurological Disorders, University of California Irvine, Irvine, CA, USA

\section{Edited by:}

Andrey V. Kuznetsov, Innsbruck

Medical University, Austria

Reviewed by:

Fadi G. Akar, Mount Sinai School of Medicine, USA

Junhui Sun, National Heart Lung and Blood Institute/National Institutes of Health, USA

\section{*Correspondence:}

Stefano L. Sensi, Molecular

Neurology Unit, Center of

Excellence on Aging, University

"G. d'Annunzio," Chieti, Italy, Via dei Vestini, 31, Chieti, 66100, Italy. e-mail:ssensi@uci.edu
Huntington's disease (HD) is a neurodegenerative condition characterized by severe neuronal loss in the cortex and striatum that leads to motor and behavioral deficits. Excitotoxicity is thought to be involved in HD and several studies have indicated that NMDA receptor (NMDAR) overactivation can play a role in the selective neuronal loss found in HD. Interestingly, a small subset of striatal neurons (less than $1 \%$ of the overall population) is found to be spared in post-mortem HD brains. These neurons are medium-sized aspiny interneurons that highly express the neuronal isoform of nitric oxide synthase (nNOS). Intriguingly, neurons expressing large amounts of nNOS [hereafter indicated as nNOS(+) neurons] show reduced vulnerability to NMDAR-mediated excitotoxicity. Mechanisms underlying this reduced vulnerability are still largely unknown and may shed some light on pathogenic mechanisms involved in HD. One untested possibility is that nNOS(+) neurons possess fewer or less functioning NMDARs. Employing single cell calcium imaging we challenged this hypothesis and found that cultured striatal nNOS(+) neurons show NMDAR-evoked responses that are identical to the ones observed in the overall population of neurons that express lower levels of nNOS [nNOS(-) neurons]. NMDAR-dependent deregulation of intraneuronal $\mathrm{Ca}^{2+}$ is known to generate high levels of reactive oxygen species of mitochondrial origin (mt-ROS), a crucial step in the excitotoxic cascade. With confocal imaging and dihydrorhodamine (DHR; a ROS-sensitive probe) we compared mt-ROS levels generated by NMDAR activation in $\mathrm{nNOS}(+)$ and $(-)$ cultured striatal neurons. DHR experiments revealed that $\mathrm{nNOS}(+)$ neurons failed to produce significant amounts of mt-ROS in response to NMDA exposure, thereby providing a potential mechanism for their reduced vulnerability to excitotoxicity and decreased vulnerability in HD.

Keywords: NADPH diaphorase, excitotoxicity, reactive oxygen species, nitric oxide synthase, Huntington's disease

\section{INTRODUCTION}

Excitotoxicity is a major pathogenic component of several neurodegenerative disorders, including Alzheimer's disease, Parkinson's disease, amyotrophic lateral sclerosis, and Huntington's disease (HD) (Choi, 2005; Lau and Tymianski, 2010; Spalloni et al., 2013). HD is an autosomal dominant neurodegenerative condition characterized by severe behavioral, cognitive, and movement disorders (Ross and Tabrizi, 2011). Inheritance of the huntingtin $(\mathrm{Htt})$ protein showing a pathogenic expansion of a glutamine stretch (polyQ repeats $>35$ ) (Macdonald et al., 1993) leads to massive cortical and striatal neuronal loss (Halliday et al., 1998; Cattaneo et al., 2005; Guo et al., 2012). Reasons for this sub-regional selectivity of the neurodegenerative process are not completely understood, although several mechanisms have been proposed. In that respect, evidence indicates that polyQ Htt promotes HD pathology through deregulation of vesicle trafficking (Difiglia et al., 1995; Qin et al., 2004), alteration of BDNF transport (Gauthier et al., 2004), disruption of microtubules (Trushina et al., 2003), interference with NMDA receptor (NMDAR) and synaptic activity (Zeron et al., 2004) as well as disruption of mitochondrial functioning and morphology (Rockabrand et al., 2007; Costa and Scorrano, 2012). 
Excitotoxicity is a phenomenon driven by excessive synaptic accumulation of glutamate and associated with dysregulation of intraneuronal $\mathrm{Ca}^{2+}\left(\left[\mathrm{Ca}^{2+}\right]_{\mathrm{i}}\right)$ homeostasis (Choi, 2005). A major feature of excitotoxicity is the NMDAR/Ca ${ }^{2+}$-dependent enhanced generation of nitric oxide (NO) and other reactive oxygen species (ROS) [reviewed in Forder and Tymianski (2009) and Szydlowska and Tymianski (2010)].

A subpopulation of striatal neurons (accounting for less than $1 \%$ of the overall population) expresses high levels of the enzyme nicotinamide adenine dinucleotide phosphatediaphorase (NADPH-d) that is the neuronal isoform of nitric oxide synthase (nNOS) (Hope et al., 1991). Intriguingly, these neurons expressing large amount of nNOS [hereafter called nNOS(+) neurons] are not affected by NMDAR-dependent toxicity and spared in the striatum of HD patients (Ferrante et al., 1985; Koh et al., 1986; Koh and Choi, 1988). The molecular and biochemical determinants of this decreased vulnerability are still not completely known. A possible simple explanation for the phenomenon is that $\operatorname{nNOS}(+)$ neurons have fewer or less active NMDARs. To test this hypothesis, we employed single cell $\mathrm{Ca}^{2+}$ imaging and assessed differences in NMDAR-evoked rises in $\left[\mathrm{Ca}^{2+}\right]_{\mathrm{i}}$ in striatal cultures, and compared responses obtained in $\mathrm{nNOS}(+)$ and the overall population of striatal neurons that express lower levels of the enzyme and hereafter called nNOS $(-)$ neurons.

Mitochondrial function is critical to maintaining $\left[\mathrm{Ca}^{2+}\right]_{\mathrm{i}}$ homeostasis (Pizzo et al., 2012). Mutant Htt promotes mitochondrial dysfunction and we have previously shown that specific $\mathrm{Htt}$ domains are crucial to drive its mitochondrial localization and promote dysfunction (Rockabrand et al., 2007).

$\mathrm{Ca}^{2+}$ overload in mitochondria promotes generation of free radicals in the organelles (Dugan et al., 1995; Reynolds and Hastings, 1995), a step that has been shown to be instrumental in the initiation of the excitotoxic cascade (White and Reynolds, 1995, 1996; Nicholls and Budd, 1998; Stout et al., 1998; Nicholls and Budd, 2000; Nicholls and Ward, 2000; Votyakova and Reynolds, 2001; Nicholls, 2002, 2004; Nicholls et al., 2003, 2007; Rintoul et al., 2003; Reynolds et al., 2004; Vesce et al., 2004; Votyakova and Reynolds, 2005). Thus, we also tested whether exposure to NMDA in striatal cultures generated different levels of mitochondrial ROS (mt-ROS) in nNOS $(+)$ neurons compared to the overall population of $\mathrm{nNOS}(-)$ cells.

\section{MATERIALS AND METHODS CHEMICALS}

Tissue culture media and sera were purchased from GIBCO (Life Technologies). Fluorescent calcium (fura-2 AM, and fluo$4 \mathrm{FF} \mathrm{AM}$ ) and ROS (dihydrorhodamine, DHR) indicators were purchased from Molecular Probes (Life Technologies). All other chemicals, unless otherwise specified, were purchased from Sigma-Aldrich.

\section{NEURONAL STRIATAL CULTURES}

All the procedures involving animals were approved by the institutional Ethics Committee (Ce.S.I.) and performed accordingly to institutional guidelines and in compliance with national and international laws and policies.
Striatal cell cultures were prepared from fetal (E15 or E16) Swiss-Webster or CD1 mice. Striatal tissues were dissected in ice-cold dissecting medium and then placed in trypsin $(0.25 \%)$ for $10 \mathrm{~min}$ at $37^{\circ} \mathrm{C}$. Tissues were centrifuged, supernatant discarded, and pellet mechanically dissociated with a glass Pasteur pipette. Cells were then resuspended in plating medium containing either: (for mixed cultures) Eagle's Minimal Essential Medium (with $20 \mathrm{mM}$ glucose, $26.2 \mathrm{mM} \mathrm{NaHCO}_{3}$ ) supplemented with L-glutamine ( $2 \mathrm{mM}), 5 \%$ fetal calf serum, and $5 \%$ horse serum (Hyclone), or (for near-pure neuronal cultures) Neurobasal Medium supplemented with L-Glutamine $(0.5 \mathrm{mM})$, $5 \%$ fetal bovine serum, $5 \%$ horse serum, $1 \times \mathrm{B} 27$, and $0.2 \%$ penicillin/streptomycin.

To prepare mixed cultures, cell suspensions were diluted and plated onto an astrocytes layer on $35 \mathrm{~mm}$ culture dishes with a glass bottom (Mat-Tek). Cells were fed twice a week with a growth medium (containing 10\% horse serum, and $2 \mathrm{mM}$ L-glutamine) and after 12 days in vitro (DIV) with a serum-free medium supplemented with $2 \mathrm{mM}$ L-glutamine.

For near-pure neuronal cultures, cells suspensions were diluted and plated onto laminin/poly-DL-lysine coated glass coverslips. Three days after plating, non-neuronal cell growth was inhibited by adding $10 \mu \mathrm{M}$ of cytosine arabinofuranoside. Twice a week, $25 \%$ of the medium was replaced with equal amounts of fresh Neurobasal medium.

Striatal neurons were used between 12 to 17 DIV.

\section{IMAGING STUDIES}

$\mathrm{Ca}^{2+}$ imaging employing fura-2 was performed using a Nikon Diaphot inverted microscope equipped with a Xenon lamp, a $40 \times$ Nikon epifluorescence oil immersion objective (N.A.: 1.3), and a CCD camera (Quantex). Fluo-4FF experiments were instead performed using a Nikon Eclipse TE300 inverted microscope equipped with a Xenon lamp, a $40 \times$ Nikon epifluorescence oil immersion objective (N.A.: 1.3) and a 12-bit Orca CCD camera (Hamamatsu). DHR experiments were performed with a confocal microscope (Noran Odyssey) equipped with an argonion laser, an inverted microscope (Nikon Diaphot), and a $60 \times$ Nikon oil-immersion objective (N.A.: 1.4). Fura-2 ratios and DHR confocal images (and relative bright field images) were digitized and analyzed using Image-1 system (Universal Imaging) or Metamorph imaging software (Universal Imaging), respectively. Fluo-4FF images were acquired and analyzed with Metafluor 6.0 software (Molecular Devices).

\section{$\left[\mathrm{Ca}^{2+}\right]_{i}$ Measurements}

Striatal cultures were loaded for $30 \mathrm{~min}$ in the dark with fura-2 AM $(5 \mu \mathrm{M})$ or fluo-4FF AM $(5 \mu \mathrm{M})$ plus $0.2 \%$ Pluronic F127 in HEPES-buffered saline solution (HCSS) $(120 \mathrm{mM} \mathrm{NaCl}$, $5.4 \mathrm{mM} \mathrm{KCl}, 0.8 \mathrm{mM} \mathrm{MgCl}, 20 \mathrm{mM}$ HEPES, $15 \mathrm{mM}$ glucose, $1.8 \mathrm{mM} \mathrm{CaCl}_{2}, 10 \mathrm{mM} \mathrm{NaOH}, \mathrm{pH} 7.4$ ), washed, and incubated for further $30 \mathrm{~min}$ in HCSS. In fura-2 experiments $\left[\mathrm{Ca}^{2+}\right]_{\mathrm{i}}$ was determined using the ratio method described by Grynkiewicz et al. (1985). Fura-2 (Ex $\lambda: 340,380 \mathrm{~nm}, \operatorname{Em} \lambda: 510 \mathrm{~nm})$ calibrated values were obtained by determining $R_{\min }$ and $R_{\max }$ using: EGTA $(10 \mathrm{mM})$ and ionomycin $(10 \mu \mathrm{M})$ in $0 \mathrm{Ca}^{2+}$ buffer for $R_{\min }$, and $\mathrm{Ca}^{2+}(10 \mathrm{mM})$ with ionomycin $(10 \mu \mathrm{M})$ for $R_{\max }$. Fura-2 $K_{d}$ 
was set at $225 \mathrm{nM}$. Results are reported as mean $\left[\mathrm{Ca}^{2+}\right]_{\mathrm{i}} \mathrm{nM} \pm$ SEM. In fluo-4FF (Ex $\lambda$ : $490 \mathrm{~nm}, \operatorname{Em} \lambda$ : $510 \mathrm{~nm}$ ) fluorescence changes of each cell $\left(F_{x}\right)$ were normalized to basal fluorescence intensity $\left(F_{0}\right)$. Results are expressed as mean $F_{x} / F_{0} \pm$ SEM values. In all experiments, NMDA $(50 \mu \mathrm{M}+10 \mu \mathrm{M}$ glycine $)$ was applied for $20 \mathrm{~s}$ and then removed through a rapid perfusion system.

\section{ROS Production Measurements}

Cells were loaded with DHR $(5 \mu \mathrm{M})$ in the dark in a $37^{\circ} \mathrm{C} / 5 \%$ $\mathrm{CO}_{2}$ incubator for $30 \mathrm{~min}$ and then studied with confocal imaging. DHR was excited at $488 \mathrm{~nm}$ and emission collected at $>515 \mathrm{~nm}$. In order to minimize DHR photo-oxidation, laser beam was used to less than $5 \%$ of full power and image acquisition intervals minimized to $\leq 2 \mathrm{~s}$ every $5 \mathrm{~min}$. DHR was maintained in the buffer throughout all the imaging session to maintain probe equilibration between the inside and outside of cells.

NMDA exposure was performed by adding NMDA (50 $\mu \mathrm{M}+$ $10 \mu \mathrm{M}$ glycine) to the baseline HCSS solution for $5 \mathrm{~min}$. NMDARs activation was then halted by addition of $10 \mu \mathrm{M}$ MK-801 and neurons imaged for additional $25 \mathrm{~min}$.

\section{NADPH-DIAPHORASE STAINING}

To identify what we call nNOS $(+)$ neurons we employed the NADPH-d staining procedure (Koh et al., 1986). To that aim, after $\mathrm{Ca}^{2+}{ }_{i}$ or DHR experiments, cultures were rinsed three times in ice-cold TBS and fixed for $30 \mathrm{~min}$ at $4^{\circ} \mathrm{C}$ in $4 \%$ paraformaldehyde/0.1 M PBS buffer. After fixation, dishes were washed with TBS and staining solution applied for $1 \mathrm{~h}$ at $37^{\circ} \mathrm{C}$. NADPH-d staining solution contained: $0.1 \mathrm{mM}$ Tris/ $\mathrm{HCl}, 0.2 \%$ Triton $\mathrm{X}-100,1.2 \mathrm{mM}$ sodium azide, $0.2 \mathrm{mM}$ nitrotetrazolium blue, and $1 \mathrm{mM}$ NADPH, pH 7.2. The staining solution was removed and cultures rinsed with TBS. After staining, dishes were re-inserted in the microscope stage and fields re-matched with those previously imaged with fura-2, fluo-4FF, and DHR. nNOS $(+)$ neurons identified as NADPH-d $(+)$ under brightfield illumination were then evaluated for their responses in the imaging experiments.

\section{STATISTICAL ANALYSIS}

Grubbs' test was performed to detect outliers, the significance level was set at $\alpha=0.05$ (no $\operatorname{nNOS}(+)$ were found to be significant outliers). Statistical analysis was performed using the Student's $t$-test for unpaired data. Results were considered statistically significant at $p<0.05$.

\section{RESULTS \\ $\left[\mathrm{Ca}^{2+}\right]_{\mathrm{i}}$ RISES UPON NMDA EXPOSURE IN nNOS(+) AND (-) STRIATAL NEURONS}

In this set of experiment, we tested whether $\operatorname{nNOS}(+)$ possess functional NMDARs and evaluated NMDAR-dependent $\left[\mathrm{Ca}^{2+}\right]_{\mathrm{i}}$ increases as an indirect parameter of receptor activity. $\left[\mathrm{Ca}^{2+}\right]_{\mathrm{i}}$ rises upon NMDA exposure were investigated with single cell $\mathrm{Ca}^{2+}$ imaging. This indirect assay is the only possible way to study NMDAR activity in specific nNOS(+) neurons. A more direct approach would have been to investigate NMDAR-evoked currents with patch clamp electrophysiology. Unfortunately, this approach is highly unfeasible given the extremely low density $(<1 \%)$ of $\mathrm{nNOS}(+)$ neurons in our striatal cultures along with the absence of any suitable marker to identify these neurons when in culture, two factors making very unlikely the possibility of successfully patching on to these cells in adequate numbers.

Striatal cultures loaded with fura-2, a high affinity $\mathrm{Ca}^{2+}$ probe $\left(K_{d}=225 \mathrm{nM}\right)$, were exposed to NMDA $(50 \mu \mathrm{M}+$ $10 \mu \mathrm{M}$ glycine) and $\left[\mathrm{Ca}^{2+}\right]_{\mathrm{i}}$ elevation assessed during and after the challenge. In this set of experiments, we observed that NMDAR-dependent $\left[\mathrm{Ca}^{2+}\right]_{\mathrm{i}}$ rises occurring in $\mathrm{nNOS}(+)$ were not statistically different from those found in the overall population of nNOS $(-)$ neurons (Figures 1A,B). To dissect and possibly reveal more subtle differences in $\left[\mathrm{Ca}^{2+}\right]_{i}$ handling between $\mathrm{nNOS}(+)$ and $(-)$ neurons, we analyzed peak amplitudes, areas under the curve (an index of the overall cytosolic $\mathrm{Ca}^{2+}$ load) and recovery phase time $(\tau)$ of the $\left[\mathrm{Ca}^{2+}\right]_{\mathrm{i}}$ changes (Figures 1C-E). None of these parameters showed statistically significant differences between the two neuronal populations. Analysis of baseline $\left[\mathrm{Ca}^{2+}\right]_{i}$ levels also showed no differences between $\operatorname{nNOS}(+)$ and $(-)$ neurons at rest (data not shown).

In the fura-2 data set we noticed that some neurons showed $\left[\mathrm{Ca}^{2+}\right]_{\mathrm{i}}$ responses in the micromolar range. Fura-2 is a high affinity $\mathrm{Ca}^{2+}$ probe, a technical limitation that leads to underestimating peak $\left[\mathrm{Ca}^{2+}\right]_{\mathrm{i}}$ rises for values above $1 \mu \mathrm{M}$ (Hyrc et al., 1997). We therefore repeated the same set of experiments with the low affinity $\mathrm{Ca}^{2+}$ indicator fluo-4FF, a probe that, with a $K_{d}$ of $9.7 \mu \mathrm{M}$, is suitable for the detection of $\left[\mathrm{Ca}^{2+}\right]_{i}$ changes occurring in the $1 \mu \mathrm{M}$ to $1 \mathrm{mM}$ range. Fluo-4FF experiments confirmed that NMDAR-dependent $\left[\mathrm{Ca}^{2+}\right]_{\mathrm{i}}$ increases are identical in $\mathrm{nNOS}(+)$ and $(-)$ neurons (Figures 2A-E). Of note, different from fura-2 experiments, in the fluo-4FF data set we observed a decreased recovery time in all neurons, a phenomenon that likely reflects the lower affinity of the probe for $\mathrm{Ca}^{2+}$.

Finally, we assessed whether nNOS $(+)$ neurons show differences in $\left[\mathrm{Ca}^{2+}\right]_{\mathrm{i}}$ response via other glutamatergic ionotropic receptors (i.e., AMPARs). To that aim, fura-2 loaded striatal neurons were exposed to kainate $(50 \mu \mathrm{M})$ and $\left[\mathrm{Ca}^{2+}\right]_{\mathrm{i}}$ levels analyzed in terms of agonist-evoked peak levels as well as overall cytosolic cation loads (as indicated by evaluation of the area under the curve of fura-2 signals). Even in this set of experiments, we did not observe significant differences between $\mathrm{nNOS}(+)$ and $(-)$ striatal neurons (data not shown).

In summary, $\mathrm{Ca}^{2+}$ imaging experiments indicated that nNOS(+) neurons possess fully functional NMDARs.

\section{ROS GENERATION UPON NMDA EXPOSURE IN nNOS(+) AND (-) NEURONS}

Aberrant $\mathrm{Ca}^{2+}$ entry through NMDARs results in cytosolic $\mathrm{Ca}^{2+}$ overload, dissipation of the mitochondrial inner membrane potential, opening of the permeability transition pore, and production of mt-ROS (Rizzuto et al., 2012). ROS are major contributors to excitotoxicity (Choi, 1992a,b; Dugan and Choi, 1994; Sayre et al., 2008). As mentioned above, NADPH diaphorase is the neuronal nNOS enzyme (Hope et al., 1991). Thus, nNOS(+) neurons are likely to be chronically exposed to an intracellular 

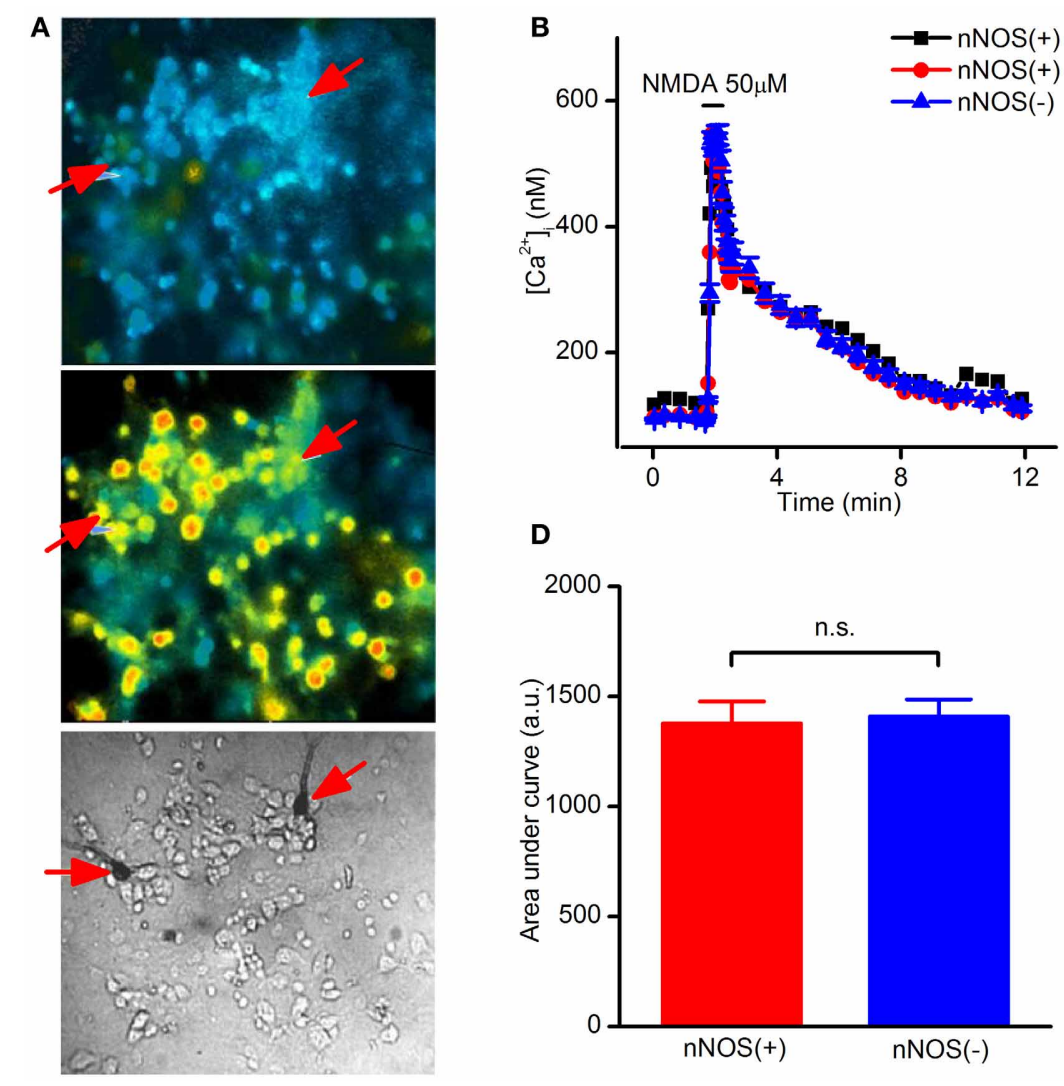

D

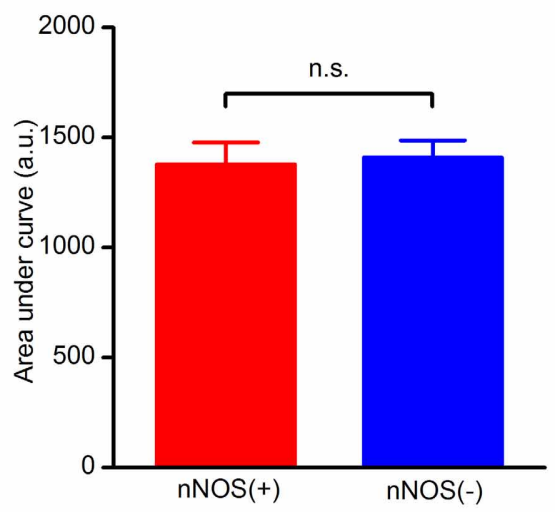

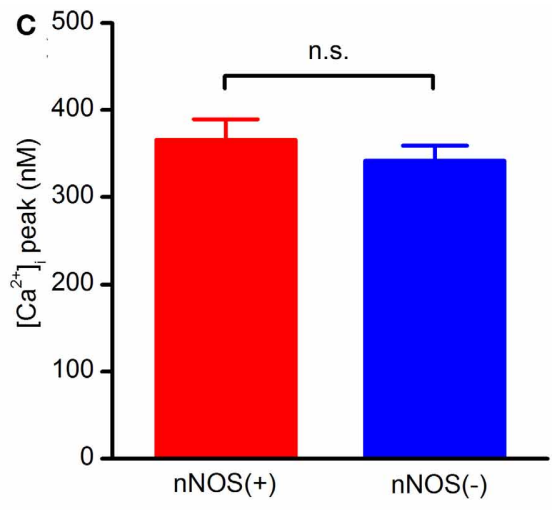

E

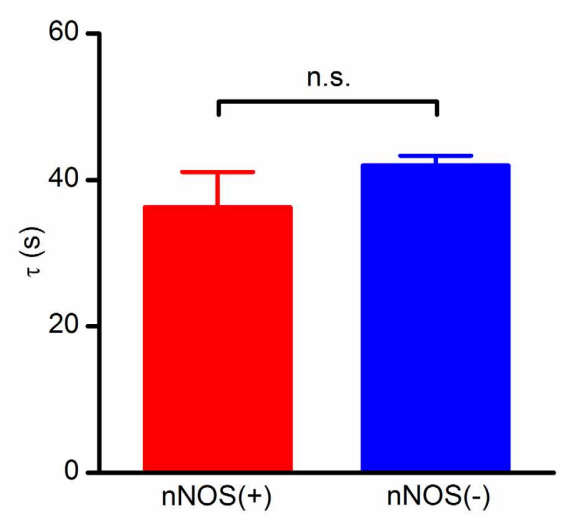

FIGURE 1 | NMDAR-dependent $\left[\mathrm{Ca}^{2+}\right]_{i}$ rises in striatal cultured neurons evaluated with a high affinity $\mathrm{Ca}^{2+}$-sensitive probe. (A) Fluorescence images of fura-2 loaded striatal neurons before (top), and upon NMDA exposure (middle). Bright field image (bottom) showing stained nNOS(+), indicated by arrows, and nNOS(-)neurons. (B) Time course of NMDAR-dependent $\left[\mathrm{Ca}^{2+}\right]_{\mathrm{i}}$ rises, traces of a single experiment $[\mathrm{NOS}(+)$ $(n=2)$ and $n N O S(-)(n=47)]$, representative of 20 independent experiments. (C) Average $\left[\mathrm{Ca}^{2+}\right]_{i}$ peak amplitude in the two neuronal populations $(p=0.42)$. (D) $\left[\mathrm{Ca}^{2+}\right]_{i}$ dynamics expressed as area under the curve $(p=0.70)$. (E) Recovery phase following NMDA exposure $(p=0.43)$. Results are expressed as mean values \pm SEM; $n N O S(+) n=22 ; n N O S(-)$ $n=560$. environment that is constantly confronted (and needs to be equipped to deal) with the presence of high NO levels.

To investigate mt-ROS levels generated by NMDAR activation, striatal neurons were first loaded with the ROS sensitive probe DHR (Henderson and Chappell, 1993) and fluorescent changes evaluated before, during, and after NMDA exposure with confocal microscopy. Native DHR is uncharged, not fluorescent, and passively diffuses through membranes. Once in the presence of ROS, DHR is oxidized to the cationic fluorescent product, rhodamine 123, allowing the investigation of mitochondrial ROS production (Dugan et al., 1995).

In this set of experiments DHR-loaded neurons, after acquisition of baseline fluorescence levels, were exposed to NMDA $(50+10 \mu \mathrm{M}$ glycine) for $5 \mathrm{~min}$, NMDAR activation was then halted by addition of the receptor antagonist (MK-801; $10 \mu \mathrm{M})$ and fluorescence changes evaluated up to $30 \mathrm{~min}$. Confocal DHR imaging revealed that NMDA application failed to promote significant fluorescence changes in $\mathrm{nNOS}(+)$ neurons while $(-)$ neurons showed significant signal rises (Figure 3).

Thus, DHR experiments revealed that NMDA exposure fails to elicit production of mt-ROS in $\mathrm{nNOS}(+)$ striatal neurons.

\section{DISCUSSION}

$\mathrm{nNOS}(+)$ neurons are spared from NMDAR-driven excitotoxicity, a phenomenon that has puzzled the field for many years (Beal et al., 1986; Koh and Choi, 1988; Kumar, 2004; El Ghazi et al., 2012). Our study offers two major findings that may help to unravel the mechanisms underlying the decreased vulnerability of these neurons and offer additional insight for their increased survival in the context of HD.

Firstly, nNOS(+) striatal neurons possess fully functional NMDARs and respond to receptor activation with $\left[\mathrm{Ca}^{2+}\right]_{\mathrm{i}}$ rises that do not differ for amplitude and temporal dynamics to the ones observed in the overall population of $\mathrm{nNOS}(-)$ neurons. Secondly, in nNOS(+) neurons, NMDAR activation does not lead to generation of mt-ROS, thereby occluding a critical downstream event of their excitotoxic cascade.

NMDAR-dependent deregulation of intraneuronal $\left[\mathrm{Ca}^{2+}\right]_{\mathrm{i}}$ levels upon prolonged or excessive glutamate exposure is the event that starts excitotoxicity (Choi, 1992a,b, 2005). Thus, the reduced vulnerability of nNOS $(+)$ neurons to excitotoxic challenges could have been easily explained by reduced expression or functioning of NMDARs in this subpopulation. 

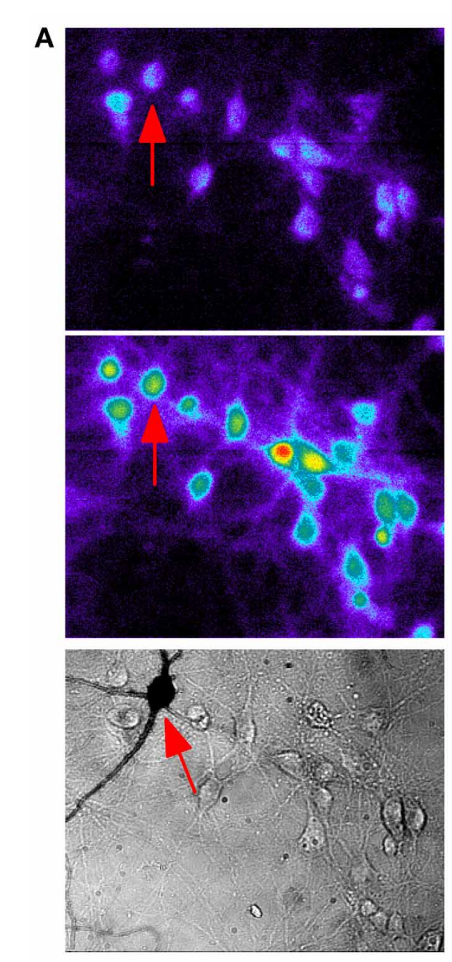

B

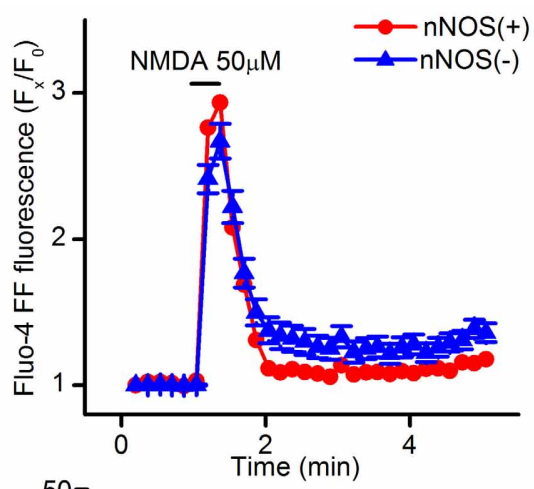

D

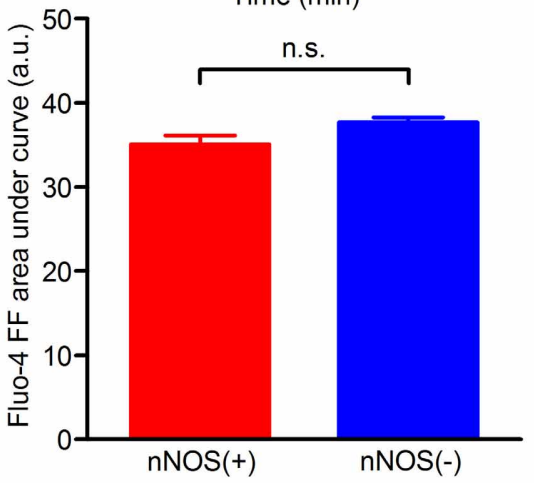

c

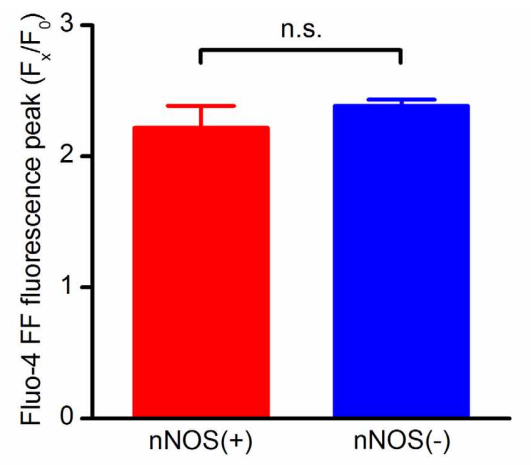

E

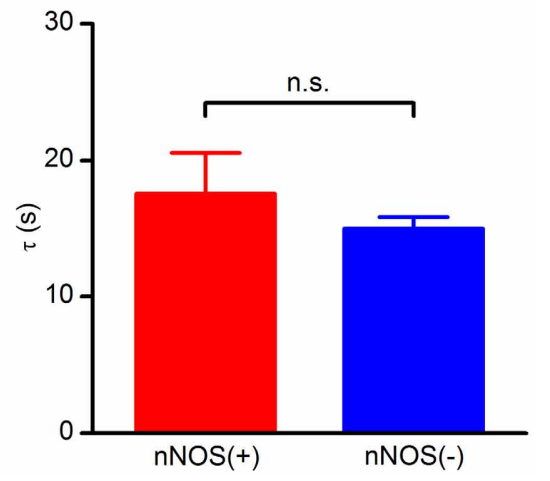

FIGURE 2 | NMDAR-dependent $\left[\mathrm{Ca}^{2+}\right]_{\mathrm{i}}$ rises in striatal cultured neurons evaluated with a low affinity $\mathrm{Ca}^{2+}$-sensitive probe. (A) Fluorescence images of fluo4-FF loaded striatal neurons before (top) and upon NMDA exposure (middle). Bright field image (bottom) showing stained nNOS(+), indicated by arrows, and nNOS(-) neurons. (B) Time course of NMDAR-dependent $\left[\mathrm{Ca}^{2+}\right]_{i}$ rises, traces of a single experiment $[\mathrm{nNOS}(+)$ $(n=1)$ and $\mathrm{nNOS}(-)(n=18)]$, representative of 12 independent experiments. (C) Average $\mathrm{Ca}_{i}^{2+}$ peak amplitude in the two neuronal populations $(p=0.36)$. (D) $\left[\mathrm{Ca}^{2+}\right]_{i}$ dynamics expressed as area under the curve $(p=0.24)$. (E) Recovery phase following NMDA exposure $(p=0.43)$. Results are expressed as mean values $\pm \mathrm{SEM} ; \mathrm{nNOS}(+) n=13 ; \mathrm{nNOS}(-)$ $n=165$
Our data lend us to hypothesize an alternative view and finally demonstrate that these neurons are most likely resistant not because they differ in number or functionality of NMDARs but, on the contrary, because they are able to set in motion protective events acting downstream to block a key step in the injurious cascade set in motion by NMDAR activation: the intraneuronal raise of mt-ROS levels.

ROS play important roles in several biological functions and are critical mediators of physiological or death signaling (Huang and McNamara, 2012; Ray et al., 2012). Upon excitotoxic conditions, NMDAR-driven production of high levels of cytosolic and mitochondrial ROS as well as nitrosative species leads to neuronal death (Floyd, 1999; Droge, 2002; Lau and Tymianski, 2010). Neurons maintain a redox homeostasis and counteract these oxidative and nitrosative hits by employing several endogenous scavenging mechanisms (Greenlund et al., 1995) like superoxide dismutases, catalases, and glutathione peroxidases.

As nNOS $(+)$ neurons express high levels of nNOS (Hope et al., 1991) and are thereby producing large amounts of NO, it is conceivable that they are primed to deal with an oxidizing intracellular environment and possess an enhanced capability to neutralize this challenge.

Landmark findings support this idea and, in fact, indicate that $\mathrm{nNOS}(+)$ express high levels of manganese superoxide dismutase
(MnSOD) (Gonzalez-Zulueta et al., 1998). MnSOD, a mitochondrial enzyme, neutralizes free radicals. The enzyme confers resistance against NMDA- and NO-mediated toxicity both in vivo and in vitro by preventing the generation of toxic peroxynitrite originating from the $\mathrm{NO}$ and $\mathrm{O}_{2}^{-}$interaction (Beckman and Koppenol, 1996; Gonzalez-Zulueta et al., 1998; Brown, 2010). Thus, our data suggest a conceptual framework in which enhanced MnSOD activity in nNOS(+) neurons might lead to the reduced ROS generation that we find upon NMDA exposure and provides potential explanation for their decreased vulnerability to NMDAR-mediated neuronal death.

Our results are providing support for the idea that mitochondria play a strategic role in the phenomenon. We acknowledge that future experiments are needed to clarify whether increased MnSOD activity is indeed play a major role in the neuroprotective pathway. We show that NMDA exposure produces $\left[\mathrm{Ca}^{2+}\right]_{\mathrm{i}}$ levels that are similar in $\operatorname{nNOS}(+)$ and $(-)$ striatal neurons. We also show that these excitotoxic $\mathrm{Ca}^{2+}$ rises fail to promote significant ROS raises in the mitochondria of $\operatorname{nNOS}(+)$ neurons. Thus, in $\mathrm{nNOS}(+)$ neurons, the behavior of mitochondria, critical actors of the excitotoxic cascade, differ.

Two major arguments support this assumption. In the $\mathrm{Ca}^{2+}$ imaging experiments we have shown that $\mathrm{nNOS}(+)$ neurons face the same NMDAR-driven $\mathrm{Ca}^{2+}$ overload. If one has to fit 

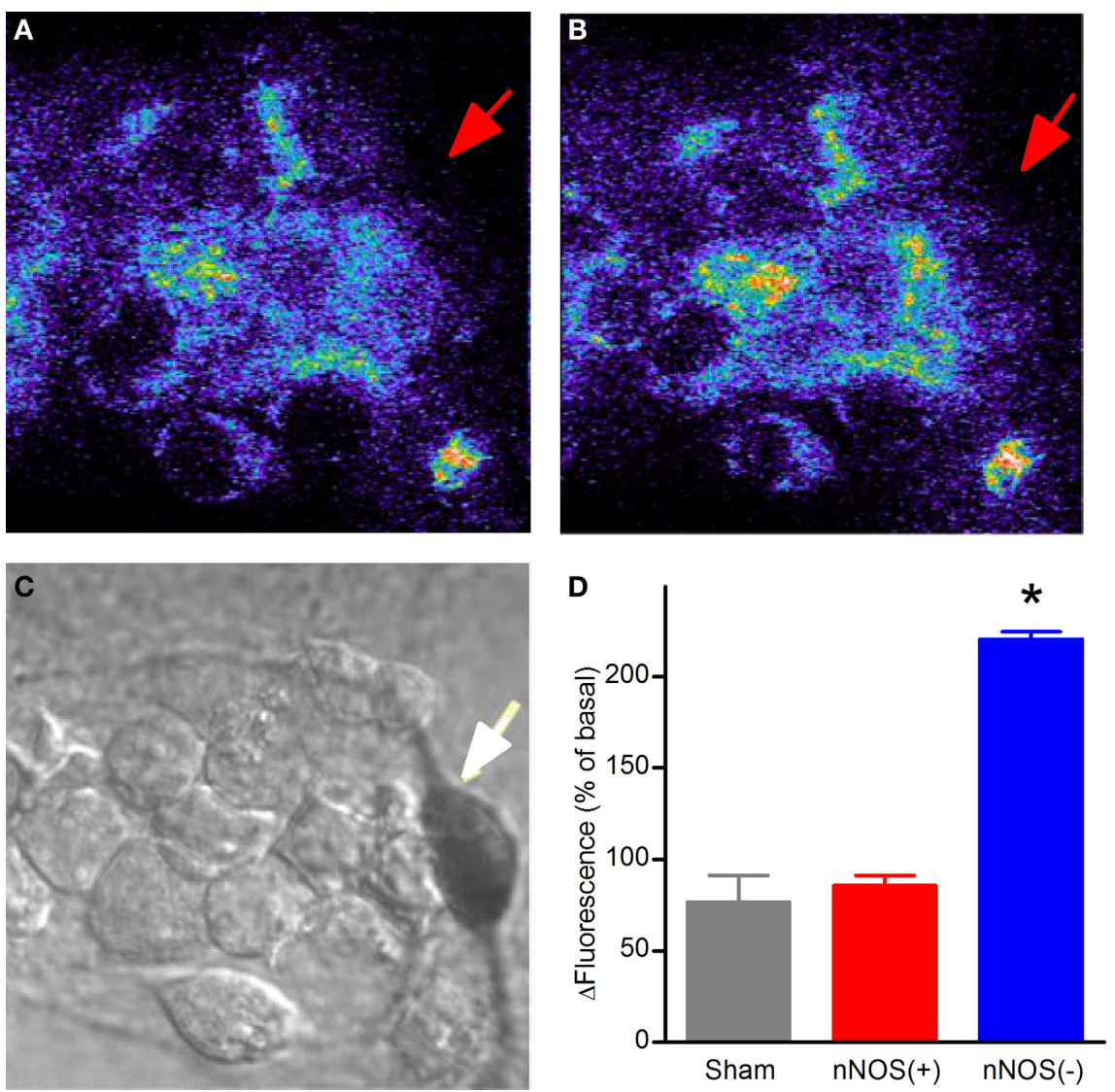

FIGURE 3 | NMDAR-dependent mitochondrial ROS production in striatal cultured neurons. (A) Confocal images of basal levels of fluorescence of DHR-loaded neurons prior to NMDA stimulation. (B) DHR fluorescence 25 min after a 5 min NMDA exposure. (C) Bright field image showing stained
nNOS $(+)$, indicated by arrow, and nNOS(-) neurons. (D) Quantification of DHR fluorescence increases 25 min after NMDA exposure $\left({ }^{*} p<0.001\right)$. Results are expressed as mean $\%$ of $F_{x} / F_{0} \pm$ SEM; nNOS( +$) n=19$; $\mathrm{nNOS}(-) n=142$ from 18 independent experiments. these data and the DHR results in a comprehensive conceptual framework, it can be hypothesized that either: (1) mitochondria of $\mathrm{nNOS}(+)$ neurons are allowing less $\mathrm{Ca}^{2+}$ uptake (thereby decreasing the overall generation of $\mathrm{Ca}^{2+}$-dependent mt-ROS) or (2) nNOS(+) neurons have developed ways to counteract the generation of mt-ROS.

When considering the first hypothesis, it should be underlined that our $\mathrm{Ca}^{2+}$ imaging experiments take in account and report changes of cation levels occurring in the cytosol, a phenomenon that is the net result of simultaneous and concerted activities of many $\mathrm{Ca}^{2+}$ homeostatic systems (Pizzo et al., 2012; Rizzuto et al., 2012). The fact that we observed similar NMDAR-driven $\mathrm{Ca}^{2+}$ loads in the two neuronal subpopulations could, in theory, be explained by compensatory mechanisms occurring in nNOS $(+)$ neurons that prevent $\mathrm{Ca}^{2+}$ overloads in mitochondria. For this mechanism to work, one has to infer that these cells may have enhanced expression and/or functioning of the plasmamembrane $\mathrm{Ca}^{2+}$-ATPases (PMCA), a key pathway for $\mathrm{Ca}^{2+}$ extrusion, a possibility that we have not tested yet. Differences in activity of the other major cellular system for $\mathrm{Ca}^{2+}$ extrusion, the plasmatic $\mathrm{Na}^{+}-\mathrm{Ca}^{2+}$ exchanger (NCX), are unlikely as excitotoxic conditions (like the one used in our setting) favor either a NCX reverse operational mode (thereby leading the exchanger to serve as pathway for $\mathrm{Ca}^{2+}$ entry) (Orrenius et al., 2003) or NCX functional blockade by calpain-mediated cleavage of the exchanger (Bano et al., 2005).

Enhanced functioning of intracellular $\mathrm{Ca}^{2+}$ stores (i.e., the endoplasmic reticulum; ER) is also unlikely to work to decrease $\mathrm{Ca}^{2+}$ rises in nNOS(+) neurons. Accordingly to the "hot spot" hypothesis proposed by Rizzuto et al. (1998), $\mathrm{Ca}^{2+}$ overload in the ER is a detrimental source for ROS generation as the cation eventually exits the ER and is taken up by mitochondria located in the ER vicinity, thereby providing the driving force for production of mt-ROS. Thus, if $\mathrm{nNOS}(+)$ neurons do overdrive the ER to maintain $\mathrm{Ca}^{2+}$ homeostasis such compensatory mechanism should be counterbalanced by enhanced levels of mt-ROS, the opposite of what we observed in our nNOS(+) neurons.

The idea that mitochondria of $\mathrm{nNOS}(+)$ sequester equal amounts of $\mathrm{Ca}^{2+}$ but respond to this hit with a decreased generation of ROS is strongly suggested by previous findings (GonzalezZulueta et al., 1998) that indicate a major role for MnSOD in promoting protection against NMDAR-mediated oxidative stress in $\operatorname{nNOS}(+)$ neurons. Within this framework, we do speculate 
that the observed reduction of ROS levels in nNOS(+) neurons may be due to higher scavenging capabilities of this neuronal subpopulation.

In summary, our data offer complementary data that substantiate a major role for mitochondria in promoting the reduced vulnerability to NMDA in nNOS(+) striatal neurons. Given the role played by polyQ Htt in affecting mitochondrial functioning,

\section{REFERENCES}

Bano, D., Young, K. W., Guerin, C. J., Lefeuvre, R., Rothwell, N. J., Naldini, L., et al. (2005). Cleavage of the plasma membrane $\mathrm{Na}+/ \mathrm{Ca} 2+$ exchanger in excitotoxicity. Cell 120, 275-285.

Beal, M. F., Kowall, N. W., Ellison, D. W., Mazurek, M. F., Swartz, K. J., and Martin, J. B. (1986). Replication of the neurochemical characteristics of Huntington's disease by quinolinic acid. Nature 321, 168-171.

Beckman, J. S., and Koppenol, W. H. (1996). Nitric oxide, superoxide, and peroxynitrite: the good, the bad, and ugly. Am. J. Physiol. 271, C1424-C1437.

Brown, G. C. (2010). Nitric oxide and neuronal death. Nitric Oxide 23, 153-165.

Cattaneo, E., Zuccato, C., and Tartari, M. (2005). Normal huntingtin function: an alternative approach to Huntington's disease. Nat. Rev. Neurosci. 6, 919-930.

Choi, D. W. (1992a). Excitotoxic cell death. J. Neurobiol. 23, 1261-1276.

Choi, D. W. (1992b). Bench to bedside: the glutamate connection. Science 258, 241-243.

Choi, D. W. (2005). Neurodegeneration: cellular defences destroyed. Nature 433, 696-698.

Costa, V., and Scorrano, L. (2012). Shaping the role of mitochondria in the pathogenesis of Huntington's disease. EMBO J. 31, 1853-1864.

Difiglia, M., Sapp, E., Chase, K., Schwarz, C., Meloni, A., Young, C., et al. (1995). Huntingtin is a cytoplasmic protein associated with vesicles in human and rat brain neurons. Neuron 14, 1075-1081.

Droge, W. (2002). Free radicals in the physiological control of cell function. Physiol. Rev. 82, 47-95.

Dugan, L. L., and Choi, D. W. (1994). Excitotoxicity, free radicals, and cell membrane changes. Ann. Neurol. 35(Suppl.), S17-S21.

Dugan, L. L., Sensi, S. L., Canzoniero, L. M., Handran, S. D., Rothman, S. M., Lin, T. S., et al. (1995). Mitochondrial production of reactive oxygen species in cortical neurons following exposure to
N-methyl-D-aspartate. J. Neurosci. 15, 6377-6388.

El Ghazi, F., Desfeux, A., Brasse-Lagnel, C., Roux, C., Lesueur, C., Mazur, D., et al. (2012). NO-dependent protective effect of VEGF against excitotoxicity on layer VI of the developing cerebral cortex. Neurobiol. Dis. 45, 871-886.

Ferrante, R. J., Kowall, N. W., Beal, M. F., Richardson, E. P. Jr., Bird, E. D., and Martin, J. B. (1985). Selective sparing of a class of striatal neurons in Huntington's disease. Science 230, 561-563.

Floyd, R. A. (1999). Antioxidants, oxidative stress, and degenerative neurological disorders. Proc. Soc. Exp. Biol. Med. 222, 236-245.

Forder, J. P., and Tymianski, M. (2009). Postsynaptic mechanisms of excitotoxicity: involvement of postsynaptic density proteins, radicals, and oxidant molecules. Neuroscience 158, 293-300.

Gauthier, L. R., Charrin, B. C., Borrell-Pages, M., Dompierre, J. P., Rangone, H., Cordelieres, F. P., et al. (2004). Huntingtin controls neurotrophic support and survival of neurons by enhancing BDNF vesicular transport along microtubules. Cell 118, 127-138.

Gonzalez-Zulueta, M., Ensz, L. M., Mukhina, G., Lebovitz, R. M., Zwacka, R. M., Engelhardt, J. F., et al. (1998). Manganese superoxide dismutase protects nNOS neurons from NMDA and nitric oxidemediated neurotoxicity. J. Neurosci. 18, 2040-2055.

Greenlund, L. J., Deckwerth, T. L., and Johnson, E. M. Jr. (1995). Superoxide dismutase delays neuronal apoptosis: a role for reactive oxygen species in programmed neuronal death. Neuron 14, 303-315.

Grynkiewicz, G., Poenie, M., and Tsien, R. Y. (1985). A new generation of $\mathrm{Ca} 2+$ indicators with greatly improved fluorescence properties. J. Biol. Chem. 260, 3440-3450.

Guo, Z., Rudow, G., Pletnikova, O., Codispoti, K. E., Orr, B. A., Crain, B. J., et al. (2012). Striatal neuronal loss correlates with clinical motor impairment in Huntington's disease. Mov. Disord. 27, 1379-1386.

this mechanism can be particularly relevant in the context of the neuronal loss occurring in the striatum of HD patients and provide targets for therapeutic intervention.

\section{ACKNOWLEDGMENTS}

Stefano L. Sensi is supported by funds from the Italian Department of Education (PRIN 2008 and 2010).

Halliday, G. M., McRitchie, D. A., Macdonald, V., Double, K. L., Trent, R. J., and McCusker, E. (1998). Regional specificity of brain atrophy in Huntington's disease. Exp. Neurol. 154, 663-672.

Henderson, L. M., and Chappell, J. B. (1993). Dihydrorhodamine 123: a fluorescent probe for superoxide generation? Eur. J. Biochem. 217, 973-980.

Hope, B. T., Michael, G. J., Knigge, K. M., and Vincent, S. R. (1991). Neuronal NADPH diaphorase is a nitric oxide synthase. Proc. Natl. Acad. Sci. U.S.A. 88, 2811-2814.

Huang, Y. Z., and McNamara, J. O. (2012). Neuroprotective effects of reactive oxygen species mediated by BDNF-independent activation of TrkB. J. Neurosci. 32, 15521-15532.

Hyrc, K., Handran, S. D., Rothman, S. M., and Goldberg, M. P. (1997). Ionized intracellular calcium concentration predicts excitotoxic neuronal death: observations with low-affinity fluorescent calcium indicators. J. Neurosci. 17, 6669-6677.

Koh, J. Y., and Choi, D. W. (1988). Vulnerability of cultured cortical neurons to damage by excitotoxins: differential susceptibility of neurons containing NADPH-diaphorase. J. Neurosci. 8, 2153-2163.

Koh, J. Y., Peters, S., and Choi, D. W. (1986). Neurons containing NADPH-diaphorase are selectively resistant to quinolinate toxicity. Science 234, 73-76.

Kumar, U. (2004). Characterization of striatal cultures with the effect of QUIN and NMDA. Neurosci. Res. 49, 29-38.

Lau, A., and Tymianski, M. (2010). Glutamate receptors, neurotoxicity and neurodegeneration. Pflugers Arch. 460, 525-542.

Macdonald, M. E., Ambrose, C. M. Duyao, M. P., Myers, R. H., Lin, C., Srinidhi, L., et al. (1993). A novel gene containing a trinucleotide repeat that is expanded and unstable on Huntington's disease chromosomes. Cell 72, 971-983.

Nicholls, D. G. (2002). Mitochondrial function and dysfunction in the cell: its relevance to aging and aging-related disease. Int. J. Biochem. Cell Biol. 34, 1372-1381.

Nicholls, D. G. (2004). Mitochondrial dysfunction and glutamate excitotoxicity studied in primary neuronal cultures. Curr. Mol. Med. 4, 149-177.

Nicholls, D. G., and Budd, S. L. (1998). Mitochondria and neuronal glutamate excitotoxicity. Biochim. Biophys. Acta 1366, 97-112.

Nicholls, D. G., and Budd, S. L. (2000). Mitochondria and neuronal survival. Physiol. Rev. 80, 315-360.

Nicholls, D. G., Johnson-Cadwell, L., Vesce, S., Jekabsons, M., and Yadava, N. (2007). Bioenergetics of mitochondria in cultured neurons and their role in glutamate excitotoxicity. J. Neurosci. Res. 85, 3206-3212.

Nicholls, D. G., Vesce, S., Kirk, L., and Chalmers, S. (2003). Interactions between mitochondrial bioenergetics and cytoplasmic calcium in cultured cerebellar granule cells. Cell Calcium 34, 407-424.

Nicholls, D. G., and Ward, M. W. (2000). Mitochondrial membrane potential and neuronal glutamate excitotoxicity: mortality and millivolts. Trends Neurosci. 23, 166-174.

Orrenius, S., Zhivotovsky, B., and Nicotera, P. (2003). Regulation of cell death: the calcium-apoptosis link. Nat. Rev. Mol. Cell Biol. 4, 552-565.

Pizzo, P., Drago, I., Filadi, R., and Pozzan, T. (2012). Mitochondrial $\mathrm{Ca}(2)(+)$ homeostasis: mechanism, role, and tissue specificities. Pflugers Arch. 464, 3-17.

Qin, Z. H., Wang, Y., Sapp, E., Cuiffo, B., Wanker, E., Hayden, M. R., et al. (2004). Huntingtin bodies sequester vesicle-associated proteins by a polyproline-dependent interaction. J. Neurosci. 24, 269-281.

Ray, P. D., Huang, B. W., and Tsuji, Y. (2012). Reactive oxygen species (ROS) homeostasis and redox regulation in cellular signaling. Cell. Signal. 24, 981-990.

Reynolds, I. J., and Hastings, T. G. (1995). Glutamate induces the production of reactive oxygen species in cultured forebrain neurons following NMDA receptor activation. J. Neurosci. 15, 3318-3327. 
Reynolds, I. J., Malaiyandi, L. M., Coash, M., and Rintoul, G. L. (2004). Mitochondrial trafficking in neurons: a key variable in neurodegeneration? J. Bioenerg. Biomembr. 36, 283-286.

Rintoul, G. L., Filiano, A. J., Brocard, J. B., Kress, G. J., and Reynolds, I. J. (2003). Glutamate decreases mitochondrial size and movement in primary forebrain neurons. J. Neurosci. 23, 7881-7888.

Rizzuto, R., De Stefani, D., Raffaello, A., and Mammucari, C. (2012). Mitochondria as sensors and regulators of calcium signalling. Nat. Rev. Mol. Cell Biol. 13, 566-578.

Rizzuto, R., Pinton, P., Carrington, W., Fay, F. S., Fogarty, K. E., Lifshitz, L. M., et al. (1998). Close contacts with the endoplasmic reticulum as determinants of mitochondrial $\mathrm{Ca} 2+$ responses. Science 280, 1763-1766.

Rockabrand, E., Slepko, N., Pantalone, A., Nukala, V. N., Kazantsev, A., Marsh, J. L., et al. (2007). The first 17 amino acids of Huntingtin modulate its sub-cellular localization, aggregation and effects on calcium homeostasis. Hum. Mol. Genet. 16, 61-77.

Ross, C. A., and Tabrizi, S. J. (2011). Huntington's disease: from molecular pathogenesis to clinical treatment. Lancet Neurol. 10, 83-98.

Sayre, L. M., Perry, G., and Smith, M. A. (2008). Oxidative stress and neurotoxicity. Chem. Res. Toxicol. 21, 172-188.

Spalloni, A., Nutini, M., and Longone, P. (2013). Role of the N-methyl-daspartate receptors complex in amyotrophic lateral sclerosis. Biochim. Biophys. Acta 1832, 312-322.

Stout, A. K., Raphael, H. M., Kanterewicz, B. I., Klann, E., and Reynolds, I. J. (1998). Glutamate-induced neuron death requires mitochondrial calcium uptake. Nat. Neurosci. 1, 366-373.

Szydlowska, K., and Tymianski, M. (2010). Calcium, ischemia and excitotoxicity. Cell Calcium 47, 122-129.

Trushina, E., Heldebrant, M. P., PerezTerzic, C. M., Bortolon, R., Kovtun, I. V., Badger, J. D. 2nd., et al. (2003). Microtubule destabilization and nuclear entry are sequential steps leading to toxicity in Huntington's disease. Proc. Natl. Acad. Sci. U.S.A. 100, 12171-12176.

Vesce, S., Kirk, L., and Nicholls, D. G. (2004). Relationships between superoxide levels and delayed calcium deregulation in cultured cerebellar granule cells exposed continuously to glutamate. J. Neurochem. 90, 683-693.

Votyakova, T. V., and Reynolds, I. J. (2001). DeltaPsi(m)-dependent and -independent production of reactive oxygen species by rat brain mitochondria. J. Neurochem. 79, 266-277.

Votyakova, T. V., and Reynolds, I. J. (2005). Ca2+-induced permeabilization promotes free radical release from rat brain mitochondria with partially inhibited complex I. J. Neurochem. 93, 526-537.

White, R. J., and Reynolds, I. J. (1995). Mitochondria and $\mathrm{Na}+/ \mathrm{Ca} 2+$ exchange buffer glutamate-induced calcium loads in cultured cortical neurons. J. Neurosci. 15, 1318-1328.

White, R. J., and Reynolds, I. J. (1996). Mitochondrial depolarization in glutamate-stimulated neurons: an early signal specific to excitotoxin exposure. J. Neurosci. 16, 5688-5697.

Zeron, M. M., Fernandes, H. B., Krebs, C., Shehadeh, J., Wellington, C. L., Leavitt, B. R., et al. (2004). Potentiation of NMDA receptormediated excitotoxicity linked with intrinsic apoptotic pathway in YAC transgenic mouse model of Huntington's disease. Mol. Cell. Neurosci. 25, 469-479.

Conflict of Interest Statement: The authors declare that the research was conducted in the absence of any commercial or financial relationships that could be construed as a potential conflict of interest.

Received: 17 January 2013; accepted: 30 April 2013; published online: 16 May 2013.

Citation: Canzoniero LMT, Granzotto A, Turetsky DM, Choi DW, Dugan LL and Sensi SL (2013) nNOS(+) striatal neurons, a subpopulation spared in Huntington's Disease, possess functional NMDA receptors but fail to generate mitochondrial ROS in response to an excitotoxic challenge. Front. Physiol. 4:112. doi: 10.3389/fphys.2013.00112

This article was submitted to Frontiers in Mitochondrial Research, a specialty of Frontiers in Physiology.

Copyright (c) 2013 Canzoniero, Granzotto, Turetsky, Choi, Dugan and Sensi. This is an open-access article distributed under the terms of the Creative Commons Attribution License, which permits use, distribution and reproduction in other forums, provided the original authors and source are credited and subject to any copyright notices concerning any third-party graphics etc. 